\title{
Microwave Power Beaming using Ka-band Radar Tethered Aerostat Program
}

\author{
Travis Haugstad ${ }^{1}$, Brian laPlante, Landon Rudy, Nicholas Watson \\ Western Technical College
}

\begin{abstract}
With electronic devices becoming smaller and more portable, they are being utilized more in science and everyday life. There must be a way to power these devices without long cables, large batteries, or other power supplies. Mobile phones and CubeSats are examples of technologies that could take advantage of wireless low-energy transfer for powering or charging devices. Our platform, containing our receiving circuitry, was suspended from a tethered aerostat. The platform was designed to receive the wireless signal emitted from a microwave transmitter, analyze the strength of the signal via a programmable microcontroller, and transmit the data back to our logging station on ground level. To receive the transmitted power in a usable state, a rectifier and multiple antennas had to be designed and built to test for reception and efficiency. The antennas designed included the Yagi-Uda and fractal-patch antennas.
\end{abstract}

\section{Introduction and Statement of Problem}

Wireless transfer of electrical power using microwaves consists of having a microwave emitter and a receiver. The emitter would be used to send the power using electromagnetic radiation within the microwave wavelength spectrum. The receiver, which would consist of an antenna tuned to a specific wavelength and an electrical circuit to convert this signal into a useful electrical signal, is used to receive the electromagnetic radiation that was sent from the emitter. The wattage produced could be used to power any electrical device.

Wireless power transfer is not an impossible feat as it has been proven possible on a larger scale using a $10 \mathrm{KW}$ transmitter. (Lan Sun Luk, Celeste, Romanacce, Chane Kuang Sang, \& Gatina, 1997) However the small-scale applications for this technology are limitless from Reducing design requirements on the space equipment such as CubeStats by removing large battery systems to move domestic uses such as charging a cellphone, or trickle charging a Tesla car while it's in the garage overnight.

The goal of the experiment is to design and test technology used to send power wirelessly from ground level using a microwave transmitter to a receiver that is secured to a platform that is suspended from an aerostat. The signal is then amplified and converted into a measurable DC voltage.

\footnotetext{
${ }^{1}$ Special thanks to WSGC for funding, education, equipment, and technical support; County of La Crosse, WI Sherriff's Department for the donation of equipment; Michael LeDocq for mentoring us throughout this entire project; and affiliate student interns: Janssen Decker, Robert Peterson, Jordan Pinski, Josh Wagner, and Lor Xiong and past members James Beier and Joel Nielsen for their hard work and dedication throughout the project; McCarty family for the use of their land to conduct our flight(s).
} 


\section{Equipment}

Aerostat and Winch Powered Spool (WPS). This project used a tethered aerostat (Fig. 1) to suspend a payload equipped with the instruments and circuitry required to carry out a specific experiment. When filled with $260 \mathrm{ft} 3$ of welding grade helium, the aerostat measured approximately $10 \mathrm{ft}$. in diameter and $7 \mathrm{ft}$. tall, and provided $10 \mathrm{lbs}$. of lift.

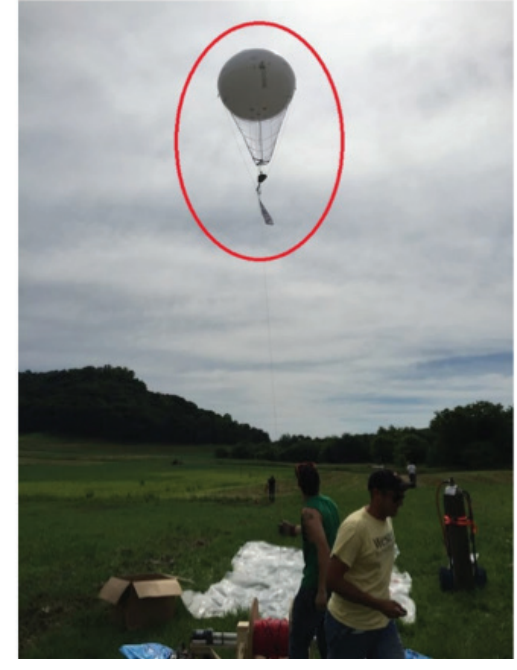

Fig. 1: Aerostat and payload package in flight

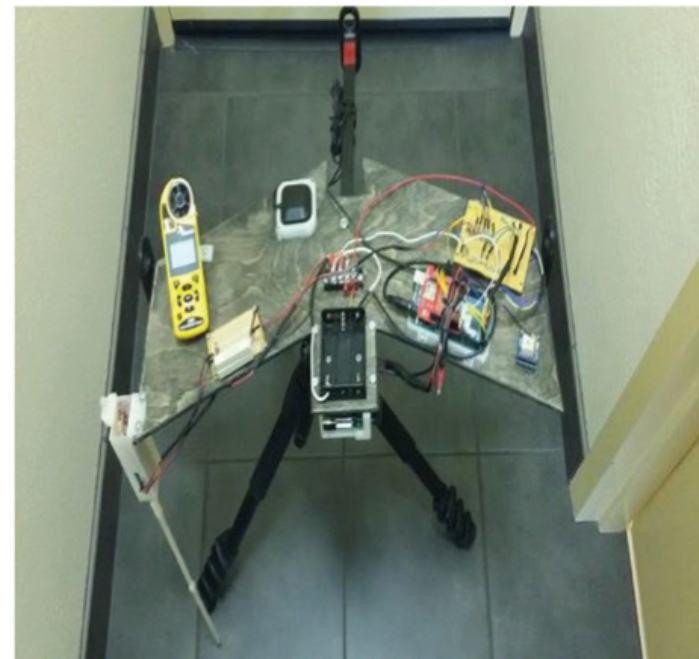

Fig. 2: Payload Platform.

Payload Platform. The payload platform (Fig. 2) was created from aircraft plywood, the platform suspends from an aerostat, and all instruments are secured to the platform. The instruments and subsystems included on the platform are an Aim Xtra flight computer, a microcontroller, and electronic circuitry that comprises of the Electrical Power and Microwave Beam subsystems.

Aim XTRA (AX) and Base Station. The AX was responsible for real time data viewing during flights and controlled a safety feature that could deflate the aerostat if the WPS failed.

Electrical Power System (EPS). This subsystem powers the microcontroller, radar detector, and AX.

Microwave Beam (MWB) Subsystem. The microwave transmitter, made by Kustom Electronics Inc., is a FALCON radar gun used by law enforcement as a tool designed to detect vehicle speed. It is used to generate $24.15 \mathrm{GHz}$ microwaves, $12-40 \mathrm{~mW}$ output power at $12^{\circ}$ beam width (Kustom Signals, Inc., 2011). The receiver is a consumer radar detector with additional circuitry that enables data collection regarding the strength of the signal received.

Arduino. This project required a microcontroller with a large supply of input and output (I/O) ports. Do to this requirement an Arduino Mega was selected. The microcontroller used to monitor battery power, radar signal strength, and data acquisition from atmospheric sensors. This data was stored to an SD card which could be downloaded later for analysis. 
Digital to Analog Converters (DAC). To allow communication with the flight computer the data had to be converted form digital logic to an analog signal where it could then be viewed at the base unit.

Antenna. Two different antenna designs were utilized to receive the microwave signal. The fractal design was made using printed circuit boards (PCB) and the Yagi-Uda antenna was built using a $3 \mathrm{D}$ printer.

\section{Subsystem Development, Theory of Operation, and Integration}

Microwave Detection Development. The accurate detection of microwave signal strength is crucial when power transfer efficiency calculations are done after the rectenna is successfully developed. It will serve as our baseline measurement. To accomplish this, a consumer radar detector was used and supporting circuitry was developed. The radar detector had a 7-segment LED display which displayed the radar signal strength detected on a scale from $0-5$. In order to convert the visual output of the LED display to a useable digital value, the voltage was measured at each of the pins to determine which LED segment was lit. The LED display is a dual inline pin package with 10 pins, 3 of which were not used to generate the display. Five volts is applied to each LED segment. When the segment of the corresponding pin is lit, there is a $2 \mathrm{~V}$ drop across the LED segment, leaving $3 \mathrm{~V}$ at the pin. Table 1 shows which LED segments are illuminated for a given signal strength. As shown in (Fig. 3), when the LED display shows a "5", LED segments a, $\mathrm{c}, \mathrm{d}, \mathrm{f}$, and $\mathrm{g}$ are lit, causing pins $3,6,7,8$, and 10 to have a low voltage.

\begin{tabular}{|c|c|c|c|c|c|}
\hline $\begin{array}{l}\text { Signal Strength on LED } \\
\text { Display }\end{array}$ & $\underline{\mathbf{5}}$ & $\underline{4}$ & $\underline{\mathbf{3}}$ & $\underline{\mathbf{2}}$ & $\underline{1}$ \\
\hline Pin 1, Segment B & High & Low & Low & Low & Low \\
\hline Pin 2 & $\mathrm{n} / \mathrm{a}$ & $\mathrm{n} / \mathrm{a}$ & $\mathrm{n} / \mathrm{a}$ & $\mathrm{n} / \mathrm{a}$ & $\mathrm{n} / \mathrm{a}$ \\
\hline Pin 3, Segment C & Low & Low & Low & High & Low \\
\hline Pin 4 & $\mathrm{n} / \mathrm{a}$ & $\mathrm{n} / \mathrm{a}$ & $\mathrm{n} / \mathrm{a}$ & $\mathrm{n} / \mathrm{a}$ & $\mathrm{n} / \mathrm{a}$ \\
\hline Pin 5 & $\mathrm{n} / \mathrm{a}$ & $\mathrm{n} / \mathrm{a}$ & $\mathrm{n} / \mathrm{a}$ & $\mathrm{n} / \mathrm{a}$ & $\mathrm{n} / \mathrm{a}$ \\
\hline Pin 6, Segment A & Low & High & Low & Low & High \\
\hline Pin 7, Segment F & Low & Low & High & High & High \\
\hline Pin 8, Segment G & Low & Low & Low & Low & High \\
\hline Pin 9, Segment E & High & High & High & Low & High \\
\hline Pin 10, Segment D & Low & High & Low & Low & High \\
\hline
\end{tabular}

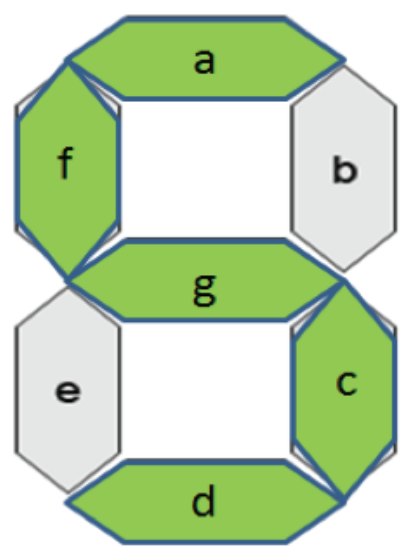

Fig 3: Table showing measures of signal strength converted to usable data and seven-segment display.

The 7 voltage values from the LED pins are then sent to analog inputs on the Arduino. The Arduino's code was written to analyze these 7 inputs and provide a $5 \mathrm{~V}$ output to multiple inputs on a voltage divider, or DAC, based on which pins are high or low. This information will be critical for future efficiency testing.

DAC.

To communicate between the Arduino and the AX a basic DAC (Fig. 4) was developed. This circuit consisted of a voltage divider and diodes. To mimic varying analog voltages five volts 
were fed into various positons on the voltage divider. By doing this the voltage divider could successfully provide a steady analog voltage to the AX which corresponded to certain data points.

Electrical Power System Development. During operation, the Arduino required 7-20 volts, the radar detector required 10-12 volts, and the AX required 3.67.4 volts. One battery pack was used to reduce weight and consisted of three lithium ion batteries in series to supply the payload with 12.6 volts peak. Zener regulator circuitry was also developed to supply the AX with $6.8 \mathrm{~V}$.

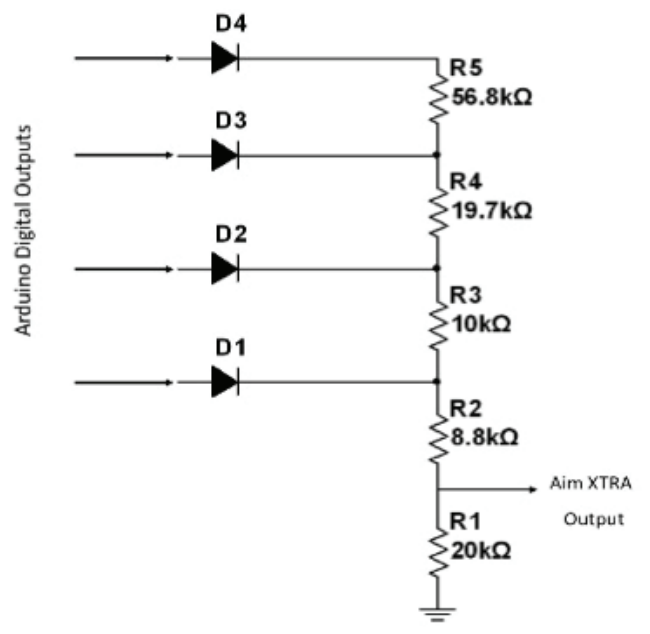

Battery life also needed to be monitored to prevent

Fig. 4: Example of a DAC used to communicate depleting the battery pack to dangerous levels. This was with the Aim Xtra. accomplished using the Arduino to control an NPN transistor circuit and voltage divider.

To conserve energy, the transistor was only forward biased every 5 seconds for 1 second. The voltage divider was used to step the battery voltage from 0 to 12.6 nominal volts down to 0 to 5 volts to be compatible with the Arduino. After the voltage was stepped down an analog input on the Arduino was then used to measure battery voltage. The measured voltage was used to make calculations, derived from the batteries' power curve that would decide which of four digital pins to turn high $(5 \mathrm{~V})$. The four pins were designated $25 \%, 50 \%, 75 \%$, and $100 \%$ battery life. The pins were fixed to a DAC which, depending on the pin that is high, will supply the AX a signal voltage from $3.1 \mathrm{~V}(100 \%)$ to $.8 \mathrm{~V}(25 \%)$. This was relayed in real time to the base unit via the AX.

Arduino Programmable Microcontroller. Weather monitoring was achieved with a Spark Fun HIH6130 chip to record humidity and temperature. These transducers would create a known voltage for a specific condition. Any changes in these voltages would indicate a change in conditions. These values were sent to an analog input on the Arduino for data logging. A Kestrel weather station ground unit was used to monitor weather conditions on the ground to enable comparison to the weather conditions on the aerostat. Any changes in conditions would indicate the conditions the microwaves will be propagating through. This data has not yet been analyzed. For the data acquisition, we used a Spark Fun SD shield. This shield was designed to be used with the Arduino Uno. Due to the need for a larger controller with more I/O pins, an Arduino Mega was implemented. This change required modification to the SD shield by clipping pins 11, 12, and 13, and using jumpers to make new connections to pins 51, 52, and 53. Signal strength, distance from the radar gun, battery power, weather conditions, and time stamps were saved to an onboard SD card in a .txt format. This data was later converted from a .txt file to a .csv file. The .csv file made enabled data viewing in an Excel format.

AX Flight Computer. The objectives of the AX were to act as the onboard flight computer, log voltage measurements of the EPS and MWB, provide GPS data including location and altitude, as well as provide a means to monitor this data in real time during flight. Some of the data, including atmospheric conditions, GPS, and altitude, were natively programmed sensors therefore making 
them somewhat intuitive to use. To obtain data regarding the EPS and MWB, the data had to be converted to voltages in the range of $0-3.1 \mathrm{~V}$, which is explained under the respective subsystems. Once these voltages were produced, they were sent to the inputs of the AX and then transmitted wirelessly to the base station. These voltages then appeared as a function of time and could be easily analyzed during flight to provide an indication of performance.

\section{Flight 1 Analysis}

Pre-Flight. While preparing to launch the aerostat, each sub-team could efficiently set up the equipment necessary to perform the experiment and collect data. Extension cords were used to power laptop chargers and a DC power supply used for the radar gun. Ground testing of the platform was performed to ensure data transmission, and the aerostat was laid out for inflation.

Once the payload had been armed and the aerostat was unpackaged, inflation began. We filled the aerostat to approximately neutral buoyancy to allow us to connect the payload platform and tether.

Flight. The flight, which was flown in 2015, was a test of various subsystems. These systems included a modified consumer radar detector and multiple DACs. The radar detector was modified to enable a live data transmission of the radar strength that the platform was receiving.

During the first flight the payload had to be brought down due to transmission issues. Due to long lengths of extension cord we were unable to diagnose the problem because $60 \mathrm{~Hz}$ noise that was introduced into our oscilloscope. It was later determined that the payload had a wiring issue caused by incorrect reinstallation of a component. To alleviate the problem in the future the platform was designed with fixed adapters and plug-ins.

\section{Post Flight Discussion}

After our first flight, we began discussing ways to achieve the goal of power transmission. To achieve this the team decided on multiple antenna designs. Along with this antenna designs a rectifier had to be developed.

\section{Antenna Design}

Fractal. Our team decided on the fractal patch antenna for the project due to its compact size and the small wavelength that was being used. The design of the fractals is based on two papers one

written by Ghaffar, Khalid, and Shamim and the other by Slovic, Jokanovic, and Kolundzija. Due to manufacturing restrictions, the various fractal patches were designed using $1 / 3$ the length of a full wave instead of the much smaller designs outlined in the papers.

To manufacture the fractal antenna PCB, acrylic paint, a laser etcher, and ferric chloride etchant were used. First the PCB was painted with acrylic paint, due to its resistance to chemical reaction, a laser etcher was then used to remove any unwanted paint after which it would be dipped into the etchant solution for 20-30 minutes. 
Yagi Uda. A small-scale Yagi Uda antenna was created to receive signal. The Yagi Uda design outputs high gain, is simple to construct, and has been producing published results since 1926. Its creation consisted of precisely trimmed copper wire and a base that was manufactured using a 3D printer, which ensured the elements were the exact distance apart to receive the microwave signal. The design of the Yagi Uda was based off an online tool that decided the dimensions (Fig. 5) that were used for the antenna. (Meserve)

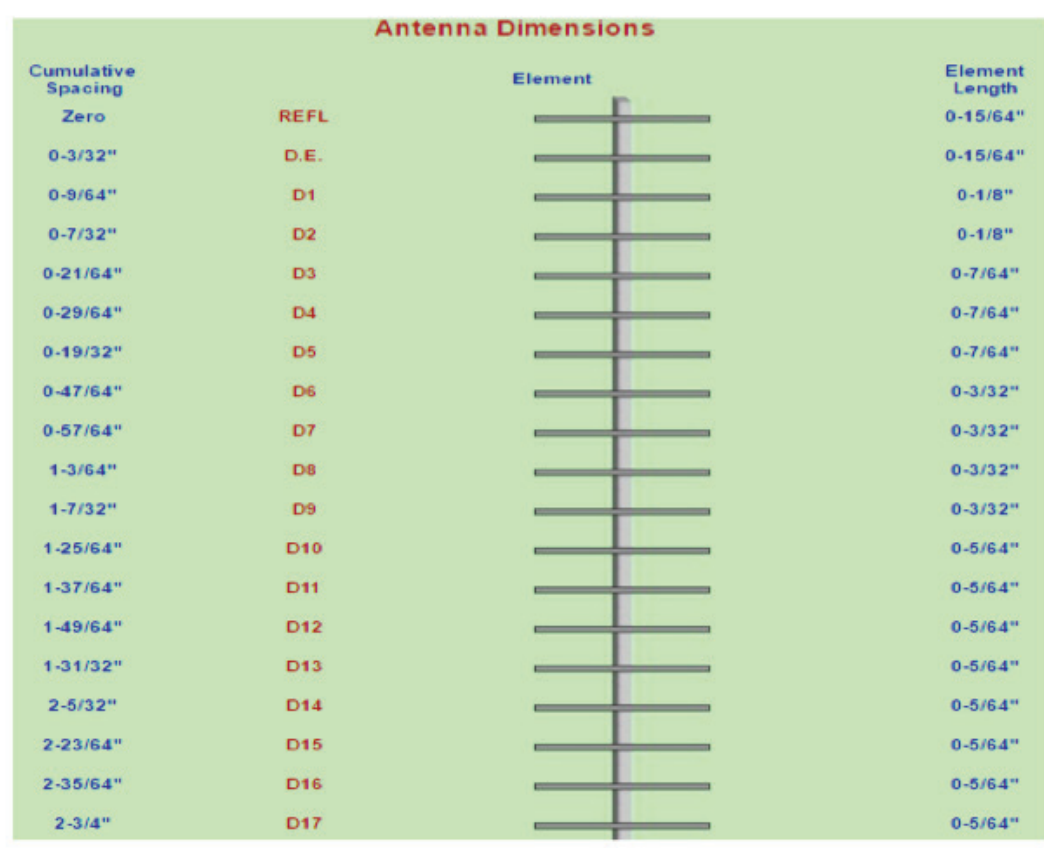

Fig. 5: Calculated lengths of and space between copper elements of antenna tunes to $24.15 \mathrm{GHz}$ signal.

Rectifier and Multiplier. The rectifier circuit (Fig. 6) that was constructed included a voltage multiplier. This is composed of a multiplier, two half wave rectifiers to operate at alternating polarities, and a resistor to test voltage. This circuit was tested at 5 volts between $5 \mathrm{kHz}-5 \mathrm{MHz}$ due to the limitations of our supply equipment. The highest frequency tested had an input voltage of 3 volts peak at $5 \mathrm{MHz}$. This yielded the highest output which was $12.20 \mathrm{~V}$ with a capacitor value of .047 micro Farads.

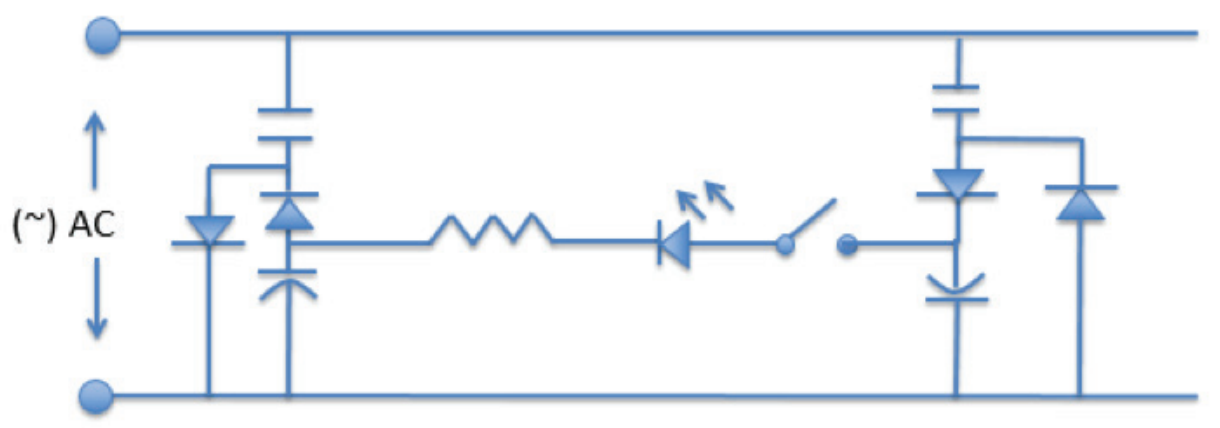

Fig. 6: Schematic diagram of a rectifying circuit. 
Antenna Challenges. Several antenna designs that could receive a $24.15 \mathrm{GHz}$ signal needed to be developed and tested. Different circuits that could rectify the $24.15 \mathrm{GHz}$ signal from said designed antenna into a DC value, and a circuit that could multiply a low voltage value, as any DC voltage that would be rectified would be miniscule at best.

All testing results were inconclusive and the problem was not immediately apparent whether it is within the antenna design or circuitry.

Next Steps. As the results proved to be inconclusive due to lack of precision instruments, such as oscilloscopes, the next steps would be to continue to research, develop, and test different antennas with instruments of a higher resolution. With the higher resolution instruments, it would be much easier to locate where the signal is being lost in the system. This would allow us to continue testing and developing off the design plan we have implemented.

Furthermore, designing antennas to receive a more common frequency of transmission could prove to be beneficial also, as most antennas researched were not designed to receive a $24.15 \mathrm{GHz}$ signal, but rather much lower frequencies. This would lose some energy that is gained from using a higher frequency signal but would allow use of easier assessable equipment and electrical components.

References

Ghaffar, Farhan A., Muhammad Umair Khalid, N. Khaled, and Atif Shamim. 24-GHz LTCC Fractal Antenna Array SoP With Integrated Fresnel Lens. Institute of Electrical and Electronics Engineers, April 2011. Web. 28 Jan. 2016.

Kustom Signals, Inc. (2011). Falcon Hand-Held Traffic Radar Operator's Manual. Lenexa, KS: Kustom Signals, Inc. Lan Sun Luk, J., Celeste, A., Romanacce, P., Chane Kuang Sang, L., \& Gatina, J. (1997). Point- to-Point Wireless Power Transportation in Reunion Island. 48th International Astronautical Congress. Turin, Italy: International Astronautical Federation.

Meserve, Martin E. "Yagi VHF." Amateur Radio Home Page. N.p., 5 June 2004. Web. 30 Dec. 2015.

Pozar, D. M. (2012). Microwave Engineer, Fourth Edition. Amherst, MA: John Wiley \& Sons, Inc.

Slovic, Marija, Branka Jokanovic, and Branko Kolundzija. High Efficiency Patch Antenna for 24 GHz Anticollision Radar. Mikrotalasna Revija, June 2006. Web. 28 Jan. 2016. 